\title{
Holguín, la más hermosa: Proyección de desarrollo del destino turístico al 2030
}

\author{
Holguin, the most beautiful: development projection of the tourist \\ destination to 2030 \\ MsC. Rosa Isidora Palao Fuentes. ${ }^{1}$, Evelina Cardet Fernández. ${ }^{2}$ \& MsC. Yanitza \\ Guerrero Rodríguez. ${ }^{3}$
}

\begin{abstract}
In the management of tourism, the combination of short-term actions, with attention to the longer-term challenges, allows a greater capacity to respond to the opportunities and threats that the sector has to face as a socio-economic activity in future scenarios, hence in Cuba today it is a priority to ensure that tourism activity has an accelerated growth that will boost the economy, based on an efficient development program. Holguin is one of the eight prioritized regions in the country for the development of international tourism. Therefore, from the indication of the Ministry of Tourism, the Development Policy of the Region was prepared in 1997, for which the diagnosis of the situation at that time in the different activities was based. Taking into account the main external threats to the destination, in this work the Tourism Development Program of Holguín 2016 - 2030 is exposed, which starts from a medium perspective scenario and expects to reach more than three hundred thousand visitors at the end of the period, which will allow achieve significant income, logically including aspects that in the last 15 years have impacted on the tourist development of the province, as well as the projections and policies to follow until 2030.
\end{abstract}

Key Words: Tourism Development, Tourist Destination, Touristic Region

\section{Resumen}

En la gestión del turismo, la combinación de las acciones inmediatas con la atención a los retos a más largo plazo, posibilita una mayor capacidad de respuesta ante las oportunidades y amenazas que, como actividad socioeconómica, ha de enfrentar el sector, pues nuevas formas de pensar que buscan mayor racionalidad propician la construcción de un nuevo proyecto de futuro autogenerador y multirrelacional. De ahí que en Cuba hoy

\footnotetext{
${ }^{1}$ Universidad de Holguín, rpalaof@uho.edu.cu

2 Delegación del Mintur Holguín, evelina@uho.edu.cu

${ }^{3}$ director@turempleo.hlg.tur.cu
} 
es una prioridad lograr que la actividad turística tenga un crecimiento acelerado que permita dinamizar la economía, sobre la base de un programa de desarrollo eficiente. Holguín es una de las ocho regiones priorizadas en el país para el desarrollo del turismo internacional. Por ello, a partir de la indicación del Ministerio de Turismo, se elaboró en 1997 la Política de Desarrollo de la Región, para lo cual se partió del diagnóstico de la situación en aquel momento en las diferentes actividades. Al tomar en consideración las principales amenazas externas al destino, en el presente trabajo se expone el Programa de Desarrollo turístico de Holguín 2016 - 2030 que parte de un escenario perspectivo medio y prevé llegar a más de quinientos mil visitantes al final del periodo, lo que permitirá alcanzar importantes ingresos, incluyendo lógicamente aspectos que en el lapso de los últimos 15 años han impactado en el desarrollo turístico de la provincia, así como las proyecciones y políticas a seguir hasta el 2030.

Palabras Clave: Desarrollo Turístico, Destino Turístico, Región Turística

\section{Introducción}

En la sociedad contemporánea se originan nuevas formas de pensar que buscan mayor racionalidad, lo que propicia la construcción de un nuevo proyecto de futuro. Las tendencias señalan las líneas de evolución que se van a confirmar en el futuro, las que se caracterizan por la internacionalización de los mercados, con nuevas exigencias; la competitividad de productos de bajo precio y máxima calidad, que incluye la coexistencia en un mismo producto múltiples servicios con atractivos naturales y valores culturales, patrimoniales e históricos; estrategias conjuntas, visión del cuidado y conservación del medioambiente; el conocimiento y el talento; flexibilidad y la creatividad; diferenciación; innovación e inventiva continua. Estos valores, unidos a la utilización de otros recursos materiales y humanos, pueden hacer de un lugar un foco de atracción permanente para el turista eventual.

La evolución acelerada que se presenta en este sector, ha provocado la necesidad de una adaptación constante de los destinos a nuevas situaciones del mercado, con nuevos gustos, y necesidades, entre otros factores. Del lado de la oferta es necesario satisfacer las expectativas de los clientes y de atender a determinados segmentos específicos del mercado lo que conlleva el aumento y diversificación de esta. Mientras por el lado de la demanda, abarca desde el crecimiento acertado de los movimientos turísticos hasta la creciente diferenciación de las motivaciones y exigencias de los turistas.

Lo expuesto con anterioridad pone de relieve la apremiante necesidad de que la gestión de los movimientos y la organización de los destinos se lleven a cabo desde una perspectiva coherente e integrada que garantice un desarrollo sostenible de la actividad.

En la gestión del turismo, la combinación de las acciones a un corto plazo, con la atención a los retos a más largo plazo, posibilita una mayor capacidad de respuesta ante las oportunidades y amenazas que ha de enfrentar el sector como actividad socioeconómica en escenarios futuros. 
El auge del turismo internacional y la propia globalización, que han permitido un conocimiento universal de los más recónditos destinos, han contribuido a destacar la importancia que tiene para el propio desarrollo del turismo y su impacto económico y social en las regiones que lo promueven, la adecuada gestión de los destinos turísticos.

Este hecho ha motivado tanto a las administraciones públicas como los propios empresarios, a buscar y aplicar metodologías y herramientas de planificación turística, así como el establecimiento de indicadores que permitan tomar las mejores decisiones sobre las estrategias a seguir para hacer ese desarrollo sostenible y lograr así una adecuada gestión.

La política del Estado Cubano para el sector turístico en la actualidad se ha ratificado y precisado, en la actualización de los Lineamientos de la Política Económica y Social del Partido y la Revolución para el período 2016-2021, aprobados por el VII Congreso del Partido Comunista de Cuba en abril de 2017, en lo referente a la Política para el Turismo, en el número 209 plantea: La actividad turística deberá tener un crecimiento acelerado que garantice la sostenibilidad y dinamice la economía, incrementando de manera sostenida los ingresos y las utilidades, diversificando los mercados emisores y segmentos de clientes, y maximizando el ingreso medio por turista.

De igual manera el lineamiento 208 relacionado con el incremento de la competitividad en los mercados turísticos establece que esta se logre a través de la diversificación de la oferta y la elevación de la calidad de los servicios. Es por ello que el turismo se ha establecido como un sector estratégico para el desarrollo del país.

El destino Holguín constituye una pieza fundamental de la maquinaria del turismo en nuestro país, pues representa uno de los destinos más grandes y hermosos de Cuba. Se destaca por la abundancia de recursos naturales, culturales, su patrimonio histórico y sus deslumbrantes playas que trascienden el espacio de las sensaciones para volverse real. Cuenta además con una fauna sorprendente, una flora con altos valores endémicos y elevaciones que contradicen las leyes de la gravedad. Es también cuna de importantes artistas de la plástica y la literatura; y sus ciudades son ricas en tradiciones y celebraciones que son muestras de la cubanía en su más alta expresión.

En la práctica el diseño e implementación de un plan de desarrollo de un destino turístico es una herramienta que permite, luego de conocer la situación que presenta, llevar a cabo una estrategia competitiva más específica y exacta, que ayude al avance sostenible del destino turístico y a su posicionamiento en el mercado internacional (Paz, 2010).

Los análisis realizados a partir del año 2010 ubicaron al destino en la etapa de Desarrollo, pero la tendencia de sus indicadores fundamentales fue hacia la consolidación del mismo. Posteriormente en el año 2013 se realizó una actualización del estudio que puso de manifiesto, una disminución del ritmo de crecimiento de sus principales indicadores, resaltando entre otras causas, el envejecimiento de la oferta.

Al considerar lo planteado con anterioridad, las exigencias y demandas de las futuras acciones de planificación y gestión en el destino, la presente investigación tiene como 
objetivo general: Actualizar el Plan de Desarrollo del destino turístico Holguín, que tome como base el uso racional de los recursos turísticos, aporte estrategias de actuación y establezca las líneas maestras para la consecución de un desarrollo turístico sostenible.

Al tener en cuenta la situación favorable o no de los factores presentes en el destino, se actualizó el Programa de Desarrollo 2016 - 2030 que parte de un escenario perspectivo medio, que prevé las cifras de visitantes a recibir al final del periodo y los correspondientes ingresos, incluye aspectos que en el lapso de los últimos 15 años han impactado en el desarrollo turístico de la provincia, así como las proyecciones y políticas a seguir hasta el 2030.

\section{Desarrollo}

"El Turismo es considerado como un fenómeno de los tiempos actuales, basado en la creciente necesidad de recuperación y cambio de ambiente, conocimientos y la apreciación de la belleza escénica, el goce del contacto con la naturaleza y es, en particular producto de la creciente fusión de las naciones y países de la sociedad humana, como resultado del desenvolvimiento del comercio, la industria y los mercados y el perfeccionamiento de los medios de transporte (García, 2013)

Lograr el aprovechamiento óptimo de las riquezas naturales y culturales es hoy en día una necesidad impostergable, y es el turismo una de las actividades que ha tomado en consideración el uso racional y adecuado de estas riquezas.

Un aspecto de mucha importancia para el estudio que se presenta, es lo referido a la gestión de los destinos turísticos. Al analizar el contexto en que hoy se desenvuelve la actividad turística a nivel mundial, con el acceso a internet, y la facilidad para el acceso a una amplia variedad de ofertas turísticas, se impone ofrecer productos turísticos innovadores que aporten nuevas posibilidades de consumo. Ello amerita de una gestión diferente en el destino en cuestión.

El destino, como sistema integrado por diversos componentes que interactúan en un espacio determinado y cuyo resultado es la oferta de servicios, no puede surgir, crecer, funcionar y evolucionar de modo anárquico; por el contrario, requiere de la necesaria coordinación entre los actores presentes en ese territorio para poder mostrar al visitante un ambiente donde prime la adecuada fluidez y armonía necesaria en toda operación turística.

Para ello se requerirá de la integración del turismo a la planificación local o regional del destino. Atender a la gestión del destino presupone actuar sobre conceptos como sostenibilidad, renovación, equilibrio, desarrollo local y regional, entre otros. Se requiere ofrecer vivencias integrales en los visitantes, siendo imprescindible actuar sobre el conjunto de la sociedad y el espacio de acogida. En definitiva, se requiere entender la planificación turística desde la perspectiva de intervención integral en el destino (Ávila y Barrado, 2005). 
En la gestión integral de los destinos, no se debe obviar la innovación y la diferenciación para ofrecer productos únicos que permitan el posicionamiento del destino; requiriendo estrategias que realcen su identidad geográfica-cultural, y convertirlo en un destino único.

La gestión integral de los destinos requiere la consideración de tres aspectos básicos: la planificación y desarrollo del destino, el desarrollo de productos y servicios turísticos, y la promoción y comunicación. Es aquí donde el Plan de Desarrollo del destino juega un papel fundamental para la integración y coordinación de los diferentes actores.

Para optimizar la gestión del destino, debe existir pluralidad en la gestión, tener en cuenta la percepción del turista respecto del destino, analizar la vulnerabilidad del mismo (manejo y prevención de conflictos y desastres), medir la rentabilidad social y económica como herramienta muy efectiva para la toma de decisiones y el análisis de la competencia (frente a otros destinos). Para poder diferenciar cualitativamente la oferta que ofrece el destino turístico, es necesario conocer qué hace la competencia.

El Plan de Desarrollo Turístico es un instrumento de gestión que contribuye a impulsar el desarrollo del sector de una localidad desde el punto de vista social y económico.

Los elementos que forman parte de la actividad turística: empresas, instituciones, profesionales, sociedad de conjunto tendrán como referencia el plan, dando sus aportaciones. Con el proceso de planificación se adquiere conciencia de que todos forman una pequeña parte de un mismo producto y por lo tanto se hace patente la necesidad de una integración y colaboración estrecha entre todos para que ese producto tenga éxito en el mercado (Rodríguez-Fariñas, 2005)

El proceso de planificación desempeña un papel básico en las posibilidades de éxito o fracaso dentro del sector turístico (destino o empresa), la ausencia de planeación genera un crecimiento desorganizado, limitando la competitividad y el aprovechamiento de los beneficios económicos y sociales. El proceso de planificación se materializa con la elaboración del Plan Estratégico, el cual es la guía básica en la aplicación de las políticas.

Para la elaboración del Plan de Desarrollo se revisaron diferentes metodologías, procedimientos y las orientaciones emanadas de la Dirección de Desarrollo del Ministerio de Turismo, las que coinciden en la realización de diagnósticos, y establecimiento de objetivos, estrategias y líneas de acción. Luego de este análisis se empleó la metodología siguiente:

El procedimiento, quedó estructurado en tres fases en forma de sistema y un total de 16 pasos, tal y como se muestra en la figura 1.

Figura 1. Procedimiento para el diseño del Plan de Desarrollo del destino Holguín 


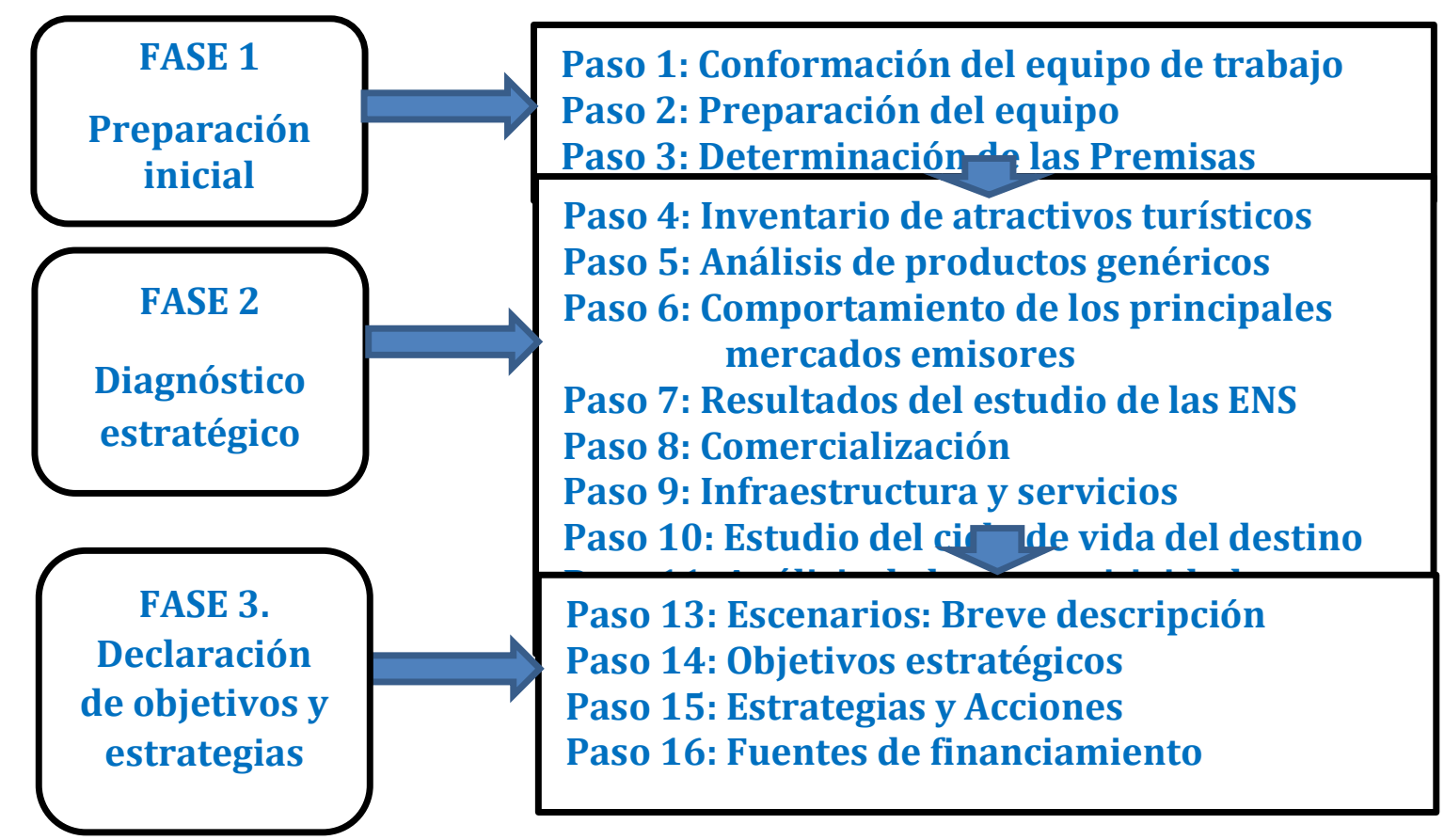

Los resultados de la aplicación del procedimiento muestran lo siguiente:

\section{Premisas del programa de desarrollo}

El desarrollo del destino turístico Holguín deberá partir de las premisas siguientes:

- Mantener la prioridad de asegurar un crecimiento acelerado que permita dinamizar la economía, sobre la base de un programa de desarrollo eficiente del destino.

- Perfeccionar las formas actuales de comercialización de la oferta utilizando las tecnologías más avanzadas de la información y las comunicaciones.

- Cada eslabón del sector en el territorio tendrá que ser eficiente, con una disminución progresiva de los costos.

- Se deberán ofrecer paquetes de servicios cada vez más integrados, diversificando y diferenciando la oferta, en correspondencia con el potencial de recursos y atractivos turísticos del territorio, priorizando el desarrollo del turismo de salud, marinas y náutica, golf, naturaleza, parques temáticos, crucerismo, historia y cultura, así como la modalidad de convenciones y eventos.

- El turismo en la provincia deberá continuar jugando un importante papel en la reanimación económica de otras ramas de la producción y el consumo de la población, aprovechando las potencialidades de todas las formas productivas a escala local.

- Mejorar la estructura de los mercados emisores y segmentos, que posibilite atenuar la estacionalidad.

- Desarrollar un turismo de paz y seguridad, preservando la calidad del medio ambiente e incrementando la competitividad del destino en los mercados. 
- Diseñar y desarrollar como parte de la iniciativa municipal, ofertas turísticas atractivas como fuente de ingreso en divisas, en especial en los municipios Holguín, Gibara, Banes, Rafael Freyre y Moa.

- Dinamizar e impulsar el desarrollo del turismo nacional mediante la creación de ofertas que posibiliten el mayor aprovechamiento de la infraestructura creada en hoteles y otros atractivos turísticos, recreativos e históricos.

- Priorizar el mantenimiento y renovación de la infraestructura turística y de apoyo, aplicando políticas que garanticen la sostenibilidad de su desarrollo.

\section{Diagnóstico estratégico}

\section{Evolución histórica del turismo en Holguín}

Holguín, fundada en 1720, se levanta hoy fraguada de historia y encantos; Colón la definió en 1492 como la tierra más hermosa....

A lo largo de su historia esta región, que no tenía el rango de provincia, contó con una cobertura constructiva de 31 hoteles, pero por diversas razones, en 1959 el territorio solamente contaba con 7 hoteles, los cuales atendidos por Turismo Popular carecían de la idoneidad requerida para ofrecer un servicio de calidad.

Igualmente, el aeropuerto del territorio no presentaba las características propias para el desarrollo de un turismo a gran escala, por no tener capacidad de acogida para los vuelos de envergadura y la recepción por esa vía era prácticamente nula.

Las playas carecían de las condiciones adecuadas para este tipo de actividad por los usos de su entorno y la no existencia de una infraestructura turística para su desenvolvimiento.

A partir de enero de 1959, los decretos y leyes que se promulgan por el Gobierno Revolucionario declaran de uso público las playas y se nacionalizan los pocos hoteles existentes, que como se señaló anteriormente no eran suficientes para desarrollar el turismo a escala superior.

Ya a partir del 20 de mayo de 1960 se construyen en Guardalavaca algunas pequeñas cabañas e instalaciones adecuadas para servir como taquillas a los bañistas, convirtiéndose en 1975 en el centro turístico que comienza a funcionar a plena capacidad ese año.

En el año 1976, con la nueva división político-administrativa quedan en el litoral norte de la nueva provincia Holguín, los municipios Gibara, Rafael Freyre y Banes, que poseen las playas con mayores potencialidades turísticas. A partir de ese momento se va fomentando el turismo nacional con la creación de condiciones habitacionales para la recepción del turismo y se edifica en la playa Guardalavaca el hotel del mismo nombre, inaugurado el 27 de Julio de 1976, por el Comandante en Jefe, con el confort idóneo para la recepción de turistas nacionales y algunos extranjeros. No es hasta el 31 de diciembre de 1980 cuando se concluye el primer hotel dentro de la ciudad, el Pernik, con categoría de tres estrellas y una capacidad de 202 habitaciones y es el primero de su tipo en todo el territorio de la provincia. 
Con la adjudicación a la provincia, por parte del Gobierno, de la condición de polo turístico priorizado, se comienza un impulso de la actividad turística de la zona con la creación de una infraestructura hotelera y de servicios en las playas del litoral norte en los municipios referidos.

Hasta el año 1989 en la provincia se desarrolló el turismo para prestar servicios a los nacionales, como sucedió en todo el país. En ese año se disponía de sólo 865 habitaciones, principalmente en la ciudad de Holguín, en Moa, un hotel en Guardalavaca y otro en Don Lino. Estos dos últimos pasaron a Cubanacán a partir de su creación en Holguín en 1987. Un elemento que enriquece este proceso de desarrollo fue el remozamiento y ampliación del aeropuerto Frank País de la provincia, con lo cual las posibilidades de arribo por esta vía aumentan de forma considerable al inaugurarse la terminal área internacional.

La cuarta provincia de mayor superficie del país, con una población de más de un millón de habitantes, al cierre del 2016 posee, como destino turístico, un total de doce polos aprobados en proceso de construcción y explotación, lo que ha permitido el crecimiento sostenido del turismo en la provincia.

\section{Estructura de la región Holguín}

La región Holguín presenta un potencial turístico de más de 64000 habitaciones y para su mejor estudio y gestión se estructura en dos subregiones, lo que se muestra en la figura 2.

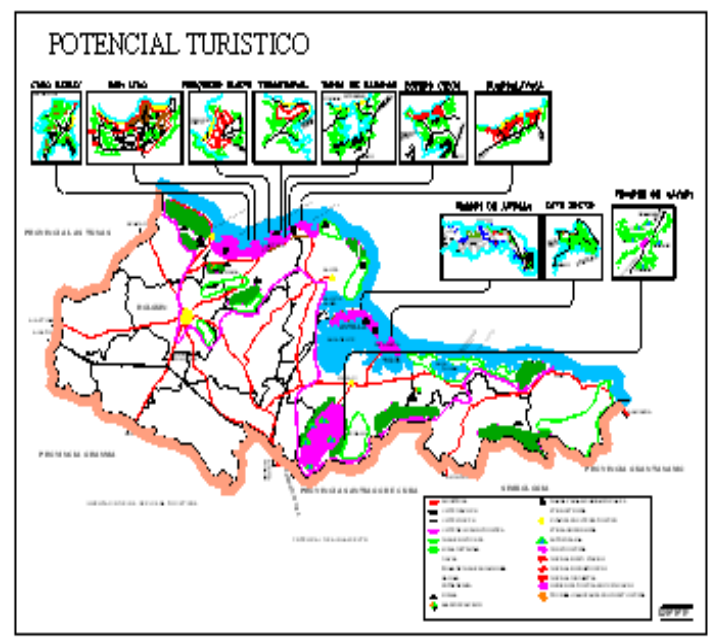

Figura 2. Potencial turístico Holguín.

Hacia el noroeste la subregión Litoral Atlántico Norte formada por los polos Guardalavaca, Estero Ciego-Caleticas(Esmeralda), Bahía de Naranjo-Yuraguanal, Pesquero Nuevo, la ciudad de Holguín, Cayo Bariay y el destino Gibara, cuyo centro histórico - declarado Monumento Nacional en 2014 - posee atractivos culturales, patrimoniales y naturales de gran singularidad. En esta región se concentra el mayor desarrollo alcanzado por el turismo en la provincia.

La otra subregión se denomina Nipe, abarca una superficie de 2495 km2 aproximadamente, se extiende desde la península del Ramón de Antilla hasta cayo Moa 
por el norte y la meseta de Pinares de Mayarí por el sur. El desarrollo actual del turismo en esta área se localiza en Cayo Saetía y la Meseta de Pinares de Mayarí.

El inventario actualizado del potencial recreativo reporta más de 220 lugares con atractivo turístico e incluye 34 ríos, varias áreas apropiadas para la inmersión, y sitios históricoculturales, 21 cuevas, 6 yacimientos de fango y aguas minero-medicinales, 12 áreas protegidas y 41 playas de excelente calidad. También en esta zona se concentra el $30 \%$ de las especies endémicas de la flora cubana y pueden observarse a más de mil especies de animales propios de la Isla, en especial aves y mariposas (López, 2016).

Para la evaluación de los recursos histórico-culturales de interés turístico se empleó una herramienta de comparación, que se fundamenta en información de entrada proveniente de la caracterización anterior: la Matriz del Índice de Atractividad, la cual puede ser de mucha utilidad en la posterior toma de decisiones.

Los más de 50 recursos histórico-culturales de interés turístico del destino se pueden agrupar en: parques, plazas, museos, centros culturales, restaurantes y cafeterías, otros lugares de interés y recursos intangibles (fiestas, eventos, tradiciones).

\section{Evaluación de los productos turísticos genéricos del destino}

Se realizó la valoración general cualitativa de los seis productos genéricos que se considera pueden diferenciar al destino Holguín: Salud, playa, náutica, naturaleza, ciudad y eventos; a partir del establecimiento de las cinco variables que más abajo se presentan y para cada de las cuales se definieron un conjunto de indicadores:

- $\quad$ Oportunidades del mercado

- Oferta de bienes y servicios que integran el producto

- Sostenibilidad

- Distribución - Comunicación

- Infraestructuras y servicios generales

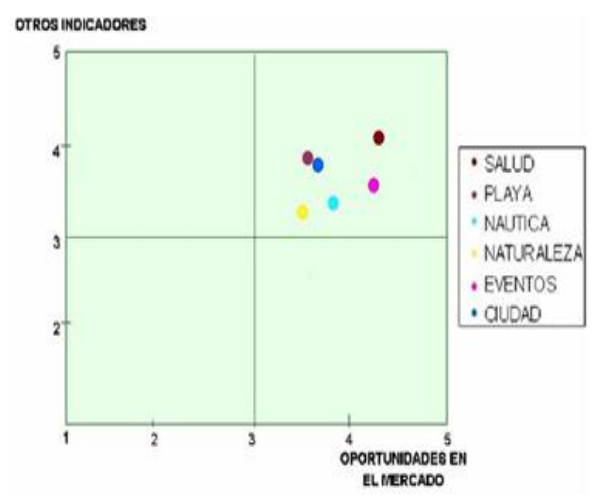

Figura 3 Resumen del diagnóstico de productos

El análisis del comportamiento de las variables e indicadores antes relacionados, para cada uno de los productos estudiados permite concluir que todos tienen oportunidades 
para su desarrollo, aunque se pueden realizar acciones que conduzcan a la mejora del producto naturaleza, aprovechando las potencialidades que tiene y las fortalezas con que cuenta, lo cual lógicamente implicará una mejora de la oferta. En la figura 3 se muestra el resumen del diagnóstico de los productos genéricos

Los principales problemas identificados fueron:

1- No existen estrategias de desarrollo de productos ante el nivel de repitencia y el envejecimiento de la oferta del destino.

2- Aunque existe demanda de los clientes por los eventos que se desarrollan en el destino, especialmente los culturales, algunos únicos de su tipo en el país, no se promueven lo suficiente y no son debidamente aprovechados por los gestores del turismo.

3- Es insuficiente la comunicación comercial en torno a todos los productos estudiados, especialmente resaltan la ausencia de materiales informativos y la bajísima presencia en internet

4- El producto ciudad tiene pocos valores añadidos a pesar de existir un considerable potencial para ello.

5- Es insuficiente el desarrollo de la actividad extra hotelera, en particular la variedad en la recreación.

6- Limitada variedad y calidad de la oferta gastronómica.

\section{Comercialización}

\section{Mezcla de los principales mercados emisores. Cuotas y su evolución}

Desde el año 1989, en que se realizó un diagnóstico de la estructura de los mercados emisores del destino y como parte de la Política de Desarrollo, se valoró que Canadá, Alemania y Reino Unido constituían los principales mercados emisores al destino.

En el análisis del comportamiento de los mercados emisores, realizado en el 2018 sobresale Canadá, alcanzando una cuota del $41.7 \%$, decreciendo un 12.2\%, Estados Unidos, quien aporta el $29.3 \%$ de los arribos, crece un 19.4\%, Inglaterra aporta el $11.7 \%$, disminuye respecto al 2017 un 5.6\%, Alemania aporta el 6.6\%, decreciendo un $21.0 \%$ y Rusia, mercado que con un año de operación en el destino, aporta un 3.6\%.Vale destacar que el mercado nacional ocupa un lugar importante en el destino, siendo en el verano, el tercer mercado emisor.

Al cierre del 2019 arribaron al destino 352729 visitantes extranjeros, con una disminución de un $0,5 \%$ con respecto al año anterior. Al descontar los que no representan alojamiento para las instalaciones hoteleras del destino, la disminución de visitantes fue del $4,7 \%$.

Canadá se mantiene como principal emisor aportando el $42 \%$ del mercado extranjero, finalizando con un incremento del 1\%. En el año decreció la presencia de clientes de Alemania, Italia, Holanda y Reino Unido; este último decreció un 39,3\% motivado por la quiebra del turoperador inglés Thomas Cook. 
Rusia con dos años de operación en el destino aporta un 5,3\%, incorporando una nueva ruta desde Moscú. El mercado nacional creció sus estancias en un 21,7\%.

A pesar de la incidencia favorable del mercado ruso y el nacional, continúa dependiendo el destino fundamentalmente del mercado canadiense.

\section{Estacionalidad}

En el destino, con un predominio de la modalidad Sol y Playa, existe una marcada estacionalidad, condicionada por las operaciones aéreas de los TTOO. Al ser el mercado canadiense el de mayor presencia, se acentúa este comportamiento.

En el 2019 se alojaron en las instalaciones turísticas del destino 2575695 turistas-días, creciendo un $0,2 \%$. Las estancias del mercado internacional decrecieron un $3,2 \%$ y el mercado nacional experimentó un crecimiento de más del $21 \%$, con una estancia media de 5,7 días.

Esta situación aconseja implementar estrategias que permitan una mayor linealidad en la ocupación.

\section{Ocupación hotelera}

En el 2018 los hoteles alcanzan una ocupación promedio del 55.6\%, alojando 2570557 turistas días totales, de ellos 2095050 extranjeros, con una estancia media de 6.7 días, una densidad ocupacional de 2.0 clientes por habitación, con un decrecimiento de $6.9 \%$, comparado con el año anterior y en el 2019 el destino cerró con una ocupación del 51\% y una densidad ocupacional de 1,98 clientes por habitación, continuando el descenso de sus indicadores.

\section{Comercialización de opcionales}

Las opcionales en el destino son comercializadas por las cuatro agencias presentes en el mismo: Cubanacán, Havanatur, Cubatur y Gaviotatour pertenecientes al turismo y la agencia Paradiso para las opcionales culturales. En el 2018 los receptivos del Mintur comercializaron las opcionales al mercado convencional, creciendo un $10,1 \%$ respecto al año 2017, con un coeficiente de compra de 0.78 , lográndose el mismo resultado al año precedente. En el 2019 se comercializaron 119857 opcionales al mercado convencional, incidiendo de forma negativa la retirada del mercado de Reino Unido, dejando de asistirse a 10800 clientes. Se actualizaron varias opcionales dirigidas al mercado ruso para atender sus expectativas.

Entre los aspectos favorables se destaca la comercialización del mercado interno que fue superior en un $31,4 \%$, así como el acercamiento a los medios de comunicación locales y la utilización de las redes sociales.

La actividad comercial se vio afectada por la insuficiente gestión de ventas de las agencias de viajes a los clientes alojados en casas de renta del territorio.

Estudio de los resultados de las encuestas nacionales de satisfacción 
Los turistas que visitan el destino muestran un crecimiento de su satisfacción a lo largo del periodo estudiado, cuyo índice se ha mantenido por encima de 4 puntos.

En el 2019 el índice de satisfacción de clientes según Encuestas de Satisfacción Nacional de manera acumulada es de 4.19, inferior en 0,01 al período anterior. Las variables de menor puntuación, inferiores a 4,0 puntos fueron: rapidez en aeropuerto 3,94, hoteles 3,8 por la incidencia de la alimentación, tiendas de hoteles 3,78 y tiendas extra hoteleras 3,84 y en aspectos generales del destino la señalización de las vías con 3.93 y facilidades de cambio de moneda con 3,85 .

Todos los hoteles del destino presentan más del 50\% de las opiniones en los sitios con evaluaciones de excelentes y muy bien, donde se destacan el hotel Brisas Guardalavaca y el Paradisus Rio de Oro, el que resultó candidato a los premios World Travel Awards.

En los hoteles las insatisfacciones se concentran en la variedad y calidad de las comidas y en el confort de las habitaciones.

En los servicios que prestan las instalaciones extra hoteleras se presentan problemas con la variedad de las ofertas gastronómicas, las dificultades asociadas al deterioro de las embarcaciones por los años de explotación en las excursiones náuticas y la inestabilidad de productos de alta demanda en las tiendas.

\section{Infraestructura y servicios}

\section{Planta hotelera}

La región turística Holguín cuenta actualmente con una capacidad de 5520 habitaciones, de las cuales corresponden 3632 a Gaviota y 1888 al Mintur.

De la planta hotelera, el 75,1\% de las habitaciones son de categoría superior, 4 y 5 estrellas en su mayoría pertenecientes al Grupo Gaviota.

\section{Infraestructura extra hotelera y de recreación}

La red extra hotelera de la región turística se encuentra situada fundamentalmente en Guardalavaca, Estero-Ciego, Pesquero, Gibara y Holguín donde están presentes instalaciones de Palmares, Caracol, Marlin, Transtur, Gaviota, Cubanacán y las agencias de viajes. Además de las entidades turísticas, operan en esta actividad Cimex y Artex; igualmente se dispone de una amplia red de establecimientos del sector no estatal.

\section{Facilidades de transporte interno}

Para la prestación del servicio de transporte automotor en la actividad turística, la provincia cuenta con cuatro entidades, ellas son: Transtur, Rex, Transgaviota y Cubataxi. Además, desde el año 2011, se ha producido un incremento de los servicios de transporte que ofrecen los trabajadores no estatales, que ha originado bases en el aeropuerto, en el centro de la ciudad y en el polo de Guardalavaca.

\section{Infraestructura técnica}

La red vial automotora presenta como eje principal a la carretera Holguín-Guardalavaca en buen estado técnico. El vínculo con el aeropuerto internacional de Holguín se completa 
por un tramo de la Carretera Central. A nivel de la subregión existen carreteras que permiten la comunicación de los polos y atractivos turísticos con las ciudades y los núcleos urbanos importantes.

Las principales vinculaciones internacionales aéreas en esta subregión se producen por el Aeropuerto Internacional Frank País, que cuenta con una pista y dos terminales: una para vuelos internacionales con capacidad para 600 pasajeros y una nacional con capacidad para 120 pasajeros, entrando y saliendo. Al sureste del polo Guardalavaca, se localiza una pista de aviación para aviones de mediano porte, con una terminal de 60 pasajeros de capacidad instantánea; muy cerca existe un helipuerto de dos plazas en el Polo Estero Ciego.

Las instalaciones náuticas fundamentales existentes en esta subregión son la Marina Internacional Puerto de Vita con 38 atraques en funcionamiento más los servicios asociados y la Base Náutica de la Bahía de Samá, ambas instalaciones ofrecen servicio de abastecimiento de combustible.

Otras facilidades estudiadas han sido el abasto de agua, el tratamiento de los residuales sólidos y líquidos, las soluciones contra incendios, los servicios de electricidad, comunicaciones, las bases de apoyo, la fuerza de trabajo disponible, los sistemas de asentamientos humanos (SAH) de los principales municipios vinculados al turismo (Banes, Rafael Freyre, Gibara y Holguín).

\section{Estado de la contratación con las formas no estatales}

En la provincia existen 1489 trabajadores por cuenta propia en la actividad de arrendamiento de viviendas y espacios, de ellos el 51\% en CUC y el $49 \%$ en CUP, con un total de 2115 habitaciones para la renta.

Se cuenta con 728 casas de renta en CUC con 754 habitaciones con una mayor concentración en los municipios de Holguín, Banes y Freyre. De ellas, 13 casas poseen contrato con las agencias de viajes y se desarrollan las operaciones sin dificultad.

En esta actividad se desarrollan diferentes acciones, entre las que se destacan las reuniones mensuales convocadas por el Sindicato, así como acciones de capacitación y despachos mensuales con la Dirección de Trabajo. Se han procesado un total de 293 solicitudes para la renta de habitaciones, las que están en proceso de aprobación.

\section{Análisis del plan de inversiones}

Para el desarrollo del turismo en el destino, cada año se han ejecutado diferentes obras, fundamentalmente en nuevas instalaciones de alojamiento, la remodelación de otras y en las instalaciones extra hoteleras.

En el 2019 se ejecutaron 216 acciones de construcción, montaje y de equipos, cumpliéndose el plan al 95,5\%.

Las principales afectaciones se concentraron en la no entrada en tiempo de suministros, equipamiento para las obras y la reposición de equipos en las instalaciones, así como la insuficiente gestión del sistema de inversiones en la fase de preparación de las mismas. 
La información analizada permitió realizar el análisis del ciclo de vida del destino, como instrumento fundamental para encauzar las acciones que permitan desarrollar una gestión más eficiente.

\section{Estudio del ciclo de vida del destino}

El estudio del ciclo de vida del destino Holguín se inició en el año 2010 donde por orientación de la Dirección de Desarrollo del Mintur se realizó un análisis de los principales destinos de sol y playa del país, entre ellos Holguín. Los resultados de ese estudio ubicaron al destino en la etapa de Desarrollo, pero la tendencia de sus indicadores fue hacia la consolidación del mismo. Posteriormente en el año 2013 se realizó como tema de investigación de trabajo de diploma una actualización de la fase del ciclo de vida del destino en cuestión debido, entre otras razones, al envejecimiento de la oferta. Dicho estudio lo ubicó en la fase de Consolidación experimentándose una disminución del ritmo de crecimiento de sus principales indicadores.

En el año 2018 la Dirección de Desarrollo del Ministerio de Turismo solicitó una actualización del estudio del ciclo de vida del destino en concordancia con los lineamientos del Partido Comunista de Cuba referidos al turismo, para poder enfrentar los retos futuros que le depara el sector.

Los resultados obtenidos del estudio muestran que el destino Holguín se mantiene en la etapa de CONSOLIDACIÓN, ya que, a pesar del crecimiento de algunos indicadores, su ritmo ha disminuido.

Se han experimentado cambios favorables debido al crecimiento de su planta hotelera y extra hotelera, los niveles de satisfacción de los clientes, incremento de las inversiones, los arribos y los ingresos. Mientras que como aspecto negativo se encuentra la estructura de los mercados, con gran dependencia del mercado canadiense, y la estancia que ha disminuido.

Figura No. 5: Matriz de evaluación del ciclo de vida del destino turístico Holguín

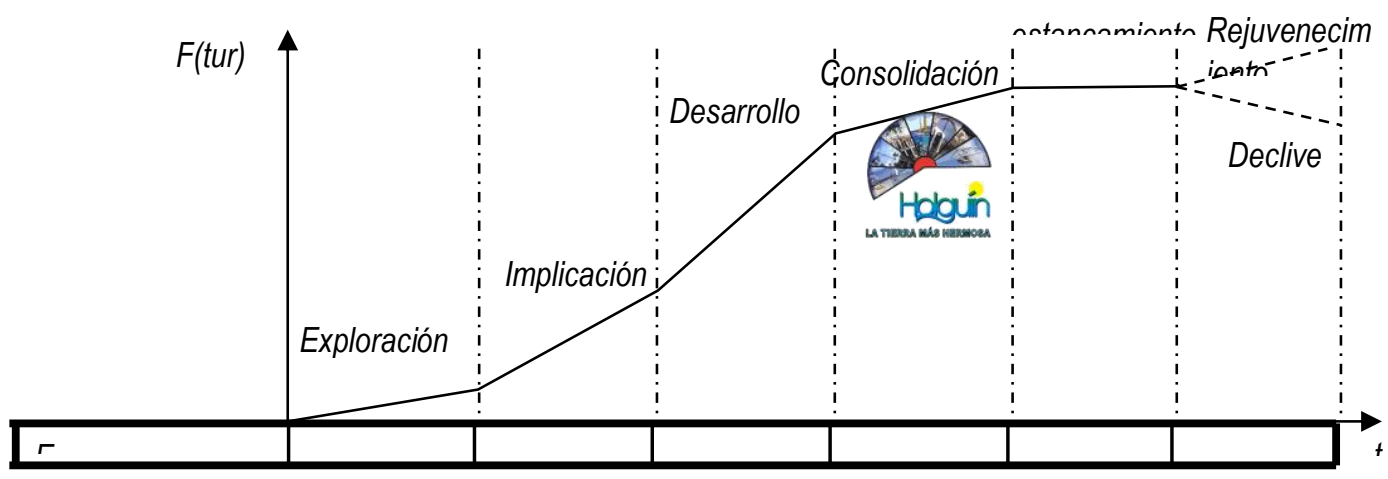

\section{Resumen del diagnóstico}

Al resumir el diagnóstico se determinaron los principales factores favorables $\mathrm{y}$ desfavorables que inciden en el desarrollo del destino. 


\section{Principales factores favorables}

- Imagen del destino.

- Altos niveles de satisfacción de los clientes, fundamentalmente en lo relacionado con la relación calidad precio.

- Asociación con partners de reconocido prestigio internacional como Sol Meliá, Fiesta Americana e Iberostar, entre otros.

- Ubicación geográfica del destino por su posicionamiento en el litoral norte del Atlántico.

- Potencialidad del sector para la diversificación de la oferta turística del destino.

- Prioridades, lineamientos, directrices y objetivos del PCC.

- Existencia de abundantes y diversos recursos turísticos de calidad.

- Tamaño y crecimiento del mercado y acceso del mercado interno a las ofertas turísticas.

- Existencia de aeropuerto internacional con todas las condiciones para recibir aviones de gran porte.

- Calificación y profesionalidad del personal.

- Posibilidades de integración de instituciones académicas y científicas para la gestión de la I+D+i.

- Desarrollo de eventos culturales únicos en el país

- El nivel de incidencia delictiva es bajo en comparación con otras regiones y países vecinos.

- Existencia de instalaciones no estatales con variados servicios turísticos de calidad con posibilidades de incorporar a la oferta del destino.

- PROBLEMAS FUNDAMENTALES QUE LIMITAN EL DESARROLLO DEL DESTINO

- El producto sol y playa representa el principal motivo de viaje al destino y es insuficiente el desarrollo de otras modalidades.

- Fuerte competencia en el área del Caribe y en otras regiones del país

- Leyes del bloqueo que impide el acceso a mercados y fuentes de abastecimientos y financiamiento

- Deterioro de parte de la planta hotelera, lo que provoca insatisfacciones en los clientes.

- Limitada oferta extra hotelera, en particular las de recreación y gastronómicas.

- Insuficiente respuesta de producción nacional a las necesidades del turismo en el destino.

- Se mantiene la insuficiente gestión de comercialización, dirigida a los principales mercados y segmentos.

- No se aprovechan todas las potencialidades de los recursos turísticos existentes para diversificar y diferenciar la oferta, fundamentalmente en las agencias de viajes.

- Existencia de mejores condiciones laborales y salariales tanto en el sector estatal como no estatal 
- Inestabilidad en la variedad y calidad de los suministros para la operación turística.

- Limitada incorporación del sector no estatal a las ofertas del destino.

- Insuficiente integración de los actores, que limita el desarrollo del turismo.

A pesar de contar con numerosas oportunidades y fortalezas, es necesario eliminar los problemas internos y atenuar las amenazas.

\section{Declaración de objetivos y estrategias}

\section{Escenario}

El Programa de Desarrollo considera múltiples factores para la construcción de los escenarios, realizando la proyección a partir de la suposición de un escenario medio caracterizado por:

- Crecimiento continuado de la emisión de turistas a nuestro país desde los actuales mercados emisores y de otros que se gestionarán, de acuerdo a la tendencia.

- Incremento de las visitas de cubanos residentes en los Estados Unidos.

- Consolidación del mercado interno como segundo emisor de turistas hacia las instalaciones del destino

- Diseño y desarrollo de ofertas turísticas atractivas, como parte de la iniciativa municipal en el territorio

- Mejoramiento del estado físico de las instalaciones turísticas

- Crecimiento de la planta hotelera e infraestructura del sector en el territorio, originado por el desarrollo de importantes inversiones.

\section{Definición de elementos estratégicos}

Se definen diferentes aspectos estratégicos necesarios para poner en marcha al Programa de Desarrollo del destino y de acuerdo con las políticas establecidas y los resultados de integración de las capacidades y la demanda.

\section{Misión}

Con playas de arenas blancas y finas, áreas de sombra, vegetación y montañas, flora y fauna diversas y ciudades que muestran un desarrollo económico en ascenso y un acervo histórico cultural, el destino turístico Holguín satisface las expectativas de sus clientes brindando un producto sostenible para lo cual cuenta con tecnología que permite ofertar servicios al turismo de sol y playa, naturaleza, náutica, buceo, salud, negocios, eventos y cultural. Conserva la zona del descubrimiento de América y la capital arqueológica de Cuba, y es el destino ideal para los que buscan distracción, intercambio cultural, paz, salud y seguridad.

\section{Visión}

Somos uno de los principales destinos turísticos de Cuba que se diferencia por la peculiar combinación de sus atractivos naturales, culturales e históricos, con una oferta diversificada que permite satisfacer a sus clientes y alcanzar un alto nivel de preferencia. 


\section{Objetivos y estrategias hasta 2030}

1. Alcanzar la excelencia del producto turístico del destino Holguín, con una oferta diversificada y diferenciada, posicionada como uno de los principales destinos de Cuba y el Caribe.

2. Garantizar los recursos humanos, cuadros y reservas calificados $y$ comprometidos, en correspondencia con la demanda del sector.

3. Alcanzar un crecimiento sostenido de los arribas de visitantes, hasta recibir no menos de 500000 turistas en el 2030.

4. Desarrollar un proceso inversionista eficiente que permita contar con un inventario de más de 13408 habitaciones, así como el desarrollo de instalaciones extra hoteleros que garantice satisfacer los niveles de demandas proyectados.

5. Garantizar una alta disponibilidad y conservación de las instalaciones ingenieras, calidad del producto turístico, permanente seguridad del servicio al cliente, un elevado rendimiento energético y la protección del medio ambiente, con una eficiente relación costo-beneficio.

6. Elevar el nivel de satisfacción de los clientes a partir del fortalecimiento de la gestión de la calidad y medioambiental, al lograr puntuaciones por encima de 4 puntos.

7. Alcanzar un alto estado político-moral, fortaleciendo la defensa, exigencia, disciplina y el control interno.

8. Implantar sistemas informáticos de gestión certificados en todas las entidades del sector en el territorio.

9. Garantizar que la actividad de investigación, desarrollo e innovación (I+D+i), propicie la planificación, búsqueda, introducción en la práctica, generalización y medición del impacto de sus resultados en la solución de los problemas concretos del sector y contribuya al desarrollo del turismo en el destino y el perfeccionamiento científico metodológico de la escuela.

\section{Principales estrategias y acciones}

1. Potenciar diferentes vías de formación y capacitación de los recursos humanos, para perfeccionar el desempeño laboral de trabajadores y mandos del territorio, logrando un mayor índice de eficacia en la operación y supervisión de los servicios.

2. Diseñar e implementar estrategias de desarrollo de mercados para consolidar los mercados ya existentes y penetrar en nuevos mercados potenciales.

3. Diseñar e implementar estrategias de desarrollo de productos, añadiendo valor a través de la ampliación de las ofertas de turismo de naturaleza, de eventos y de ciudad, fundamentalmente en los polos de Holguín, Rafael Freyre, Gibara y Antilla, aprovechando proyectos de desarrollo local.

4. Perfeccionar y ampliar la oferta de las agencias de viajes: Diseñar y lanzar al mercado nuevas opcionales, así como mejorar las existentes a través de la incorporación de los atributos que distinguen al destino (Cayo Bariay, Aldea 
Taina, Gibara, Visitas a Museos, entre otros), ofertas únicas y variadas para familias y grupos de personas, ofertas de estancias combinadas y otras de hoteles de altos estándares que generen un ingreso medio alto, productos exclusivos de Havanatur, ofertas para grandes clientes, ofertas para el mercado americano, entre otras.

5. Perfeccionar y ampliar la oferta de los prestatarios extra hoteleros (restauración, náutica, transporte, comercio) atendiendo a las características del mercado internacional y el nacional, en especial ofertas gastronómicas con el rescate de la Cocina Cubana y Regional., parques recreativos, temáticos y acuáticos, festivales náuticos en el polo Guardalavaca, programas de inserción de grupos de alta convocatoria en Centros Nocturnos, ofertas de transporte combinados, apertura de nuevos puntos náuticos, así como diferenciar y tematizar las tiendas y restaurantes

6. Ampliación de la red de ventas a través de nuevos TTOO y Agencias de Viajes, que permitan penetrar nuevos mercados.

7. Diseño e implementación de una campaña promocional dirigida al público objetivo, que apoye la estrategia de posicionamiento del destino y de la imagen Holguín como destino turístico, diferenciado y diversificado con el empleo de tecnologías de avanzada.

8. Perfeccionar el sistema de información del destino, que permita el monitoreo y análisis permanente de los principales indicadores y sirva de alerta temprana ante la manifestación o movilidad de las tendencias internacionales y sus cambios para actuar en consecuencia y oportunamente.

9. Desarrollo de programas de mantenimiento y preservación de los principales recursos turísticos del destino.

10. Potenciar la actividad de la I+D+i en la solución de los problemas de la gestión del destino y de las entidades del sector, a través de la colaboración con instituciones educativas y de investigación, en especial con el Parque Científico y Tecnológico de la Universidad de Holguín

11. Fomentar el desarrollo y las relaciones con el sector no estatal para la prestación de servicios de calidad y la ampliación de la oferta turística.

12. Potenciar el encadenamiento productivo y la participación nacional en la sustitución de importaciones, garantizando la calidad y el cumplimiento de los estándares en las ofertas turísticas.

13. Perfeccionar el proceso inversionista, en especial la fase preparatoria y el control que garantice la ampliación y renovación por mejoras de las instalaciones turísticas previstas en el plan.

14. Potenciar las inversiones a través de las fuentes de financiamiento propio y con asociaciones extranjeras.

\section{Conclusiones}


El cumplimiento del objetivo general de la presente investigación, así como la aplicación de un procedimiento para el diseño del plan de desarrollo del destino Holguín permite arribar a las conclusiones siguientes:

- El diseño del plan de desarrollo del destino turístico holguinero da respuesta a la implementación de los Lineamientos de la Política Económica y Social del Partido y la Revolución para el período 2016-2021, en lo referente a la Política para el Turismo, aprobados en el VII Congreso del Partido en abril de 2016.

- El análisis de los principales factores que inciden en el desarrollo del turismo en el destino permitió determinar las principales oportunidades y factores limitantes para elevar los niveles de competitividad del destino.

- La actualización del Plan de Desarrollo del destino Holguín 2016-2030 centra su estrategia en el desarrollo y diversificación de la oferta del destino, al desarrollar el turismo especializado, con una proyección de inversiones que garantice el crecimiento de su planta hotelera y extra hotelera.

- El Plan de Desarrollo del destino refleja las principales aspiraciones y direcciones de trabajo que permitirán alcanzar un posicionamiento superior entre los destinos del país

\section{Referencias bibliográficas}

Ávila, R., \& Barrado, D. (2005). Nuevas tendencias en el desarrollo de destinos turísticos: marcos conceptuales y operativos para su planificación y gestión. Cuadernos de Turismo, 27-43.

Centro de Estudios Turísticos. (2005). Gestión de destinos. Monografía, La Habana.

Cuba, M. (2016). Estrategias para la comunicación comercial del destino Holguín. Tesis en opción al título de Licenciada en Turismo, Universidad de Holguín, Holguín.

Delegación Mintur Holguín. (2016). Política de Desarrollo 2012-2016 del destino turístico Holguín.

Delegación Mintur Holguín. (2018). Informe de Balance Delegación de Turismo Holguín. Holguín.

Durán, E. (2013). Actualización del ciclo de vida del destino turístico Holguín. Tesis en opción al título de Licenciado en Turismo, Universidad de Holguín, Holguín.

Fernández, C., Fuentes, P., \& Pérez, M. (2011). Valoración general cualitativa de los productos genéricos existentes en el destino Holguín.

Gallegos Peralta, G. (2015). Orientaciones para la elaboración del plan de desarrollo de un destino turístico. Sernatur. 
García, A. (2013). Impacto de la crisis mundial en el turismo en América Latina y el Caribe. Retos para Cuba. Investigación Económica, 8(2), 18-26.

INFOTUR. (2018). Resultados de las encuestas de satisfacción del cliente del destino Holguín.

López, L. (2016). Diseño de una campaña publicitaria para el destino turístico Holguín. Tesis en opción al título de Licenciado en Turismo, Universidad de Holguín, Holguín.

Marrero Cruz, L. (2016). Tendencias y perspectivas del turismo cubano. Conferencia de prensa.

Organización Mundial del Turismo. (2018). Resultados del turismo internacional en 2017: los más altos en siete años.

Partido Comunista de Cuba. (2017). Documentos del 7mo Congreso del Partido. Tabloides I y II, La Habana.

Paz, R. (2010). Análisis del ciclo de vida del destino turístico Holguín. Tesis en opción al título de Licenciado en Turismo, Universidad de Holguín, Holguín.

Rodríguez Driggs, E. (2018). Análisis del ciclo de vida del destino turístico Holguín. Tesis en opción al título de Licenciado en Turismo, Universidad de Holguín, Holguín.

Rodríguez, L. (2019). Gestión del Destino Turístico y su Planificación Estratégica. Recuperado de https://www.gestiopolis.com/gestion-del-destino-turistico-y-suplanificacion-estrategica/

Rodríguez-Fariñas, R. (2005). Gestión de Destinos Turísticos. Centro de estudios turísticos Universidad de La Habana, La Habana.

Turísticos, C. d. (2005). Gestión de Destinos Turísticos. Monografía, La Habana.

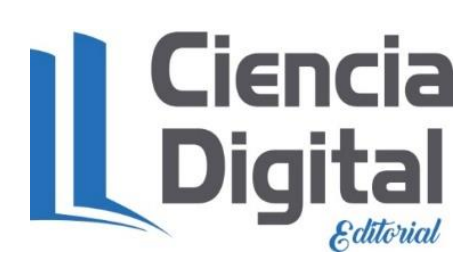




\section{PARA CITAR EL ARTÍCULO INDEXADO.}

Palao Fuentes, R. I., Cardet Fernández, E., \& Guerrero Rodríguez, Y. (2021). Holguín, la más hermosa: Proyección de desarrollo del destino turístico al 2030. Explorador Digital, 5(1), 152-172. https://doi.org/10.33262/exploradordigital.v5i1.1495

\section{¿Ciencia}

El artículo que se publica es de exclusiva responsabilidad de los autores y no necesariamente reflejan el pensamiento de la Revista Explorador Digital.

El artículo queda en propiedad de la revista y, por tanto, su publicación parcial y/o total en otro medio tiene que ser autorizado por el director de la Revista Explorador Digital.
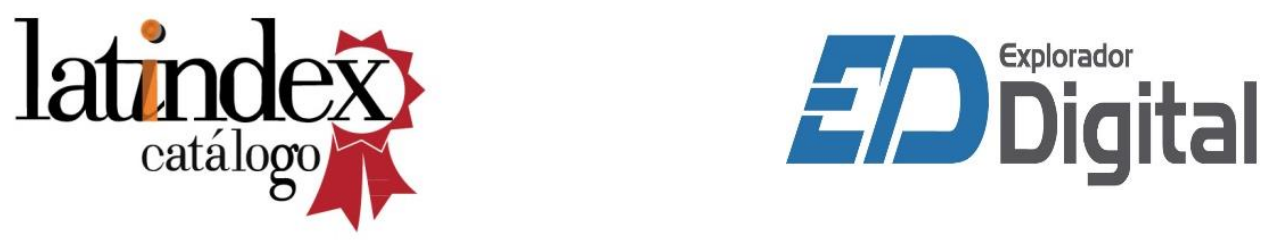